\title{
Interference in the perceived segregation of equal-luminance element-arrangement texture patterns
}

\author{
JACOB BECK \\ University of Oregon, Eugene, Oregon
}

\begin{abstract}
Perceived segregation in element-arrangement patterns composed of squares of equal size and luminance, but of two different hues, was investigated in two experiments. Element-arrangement patterns consist of two types of elements, arranged in alternating vertical stripes in the top and bottom regions and in a checkerboard pattern in the center region. Perceived segregation of the striped and checkerboard regions decreased with increasing luminance of the interspaces between the squares, a high-luminance surround, and the increased spacing of the squares. When the luminance of the horizontal interspaces was increased, the decrease in perceived segregation was greater than that when the luminance of the vertical interspaces was increased. Two explanations of the interference of the interspace luminance are discussed. One explanation is in terms of inhibitory interactions among cortical filters tuned to spatial frequency and orientation. A second explanation is in terms of interference with preattentive grouping processes.
\end{abstract}

Numerous investigators have shown that differences in two-dimensional spatial-frequency content (i.e., differences in the way textures stimulate difference of Gaussians and Gabor filters) account for how well different texture regions are perceptually segregated (Beck, Sutter, \& Ivry, 1987; Bergen \& Landy, 1991; Fogel \& Sagi, 1989; Graham, Beck, \& Sutter, 1992; Malik \& Perona, 1990; Sutter, Beck, \& Graham, 1989; Turner, 1986). The perceptual segregation of element-arrangement patterns has been shown to be in accordance with differences in spatial-frequency content when the two types of elements composing a pattern differ in either size or luminance (Graham et al., 1992; Sutter et al., 1989). Element-arrangement patterns consist of two types of elements that are arranged in alternating vertical stripes in the top and bottom regions and in a checkerboard pattern in the center region (see Figure 1). The channels that signal the difference between the striped and checkerboard regions are sensitive to the frequencies near the fundamental spatial frequency of the pattern. These channels signal the difference in the average contrast (i.e., the contrast $\times$ area) of the two squares (Sutter et al., 1989). Linear channels fail to completely explain perceived segregation, and two kinds of nonlinear processes have been proposed: a rectifying nonlinearity and an intensity-dependent nonlinearity (Graham et al., 1992).

Strong texture segregation occurs not only in terms of luminance differences, but also in terms of hue differ-

I thank Norma Graham, Ennio Mingolla, and Hans-Christoph Nothdorft for comments on an earlier version of the manuscript. Address correspondence to J. Beck, Department of Psychology, University of Oregon, Eugene, OR 97403. ences (Beck et al., 1987). Little, however, is known of the mechanisms involved when texture segregation is due to hue differences. In the two experiments reported here, the role of hue differences in segregating the striped and checkerboard regions in an elementarrangement pattern was investigated. The elementarrangement patterns were composed of equal-size squares, differing in hue. The luminances of the two hues in a pattern were equated to minimize differences in the outputs from broadband-oriented receptive fields (simple cells). Residual differences in the activation of luminance-driven mechanisms may remain, but these would not yield strong texture segregation. It should be noted that equal luminance of the squares is not a necessary condition for the present results. Ongoing experiments show that residual luminance differences are not crucial, since similar results are obtained when the luminances of the two hues in a pattern are not equated, but differ by a small amount.

Beck et al. (1987) reported that subjects failed to segregate the striped and checkerboard regions composed of red and blue squares of equal size and luminance in an element-arrangement pattern when the background luminance was high, but that perceptual segregation occurred when the background luminance was equated with the luminance of the squares. Observations indicated that perceived segregation improved further when the background luminance was lowered to a level that was below that of the squares. These results indicate that a high-luminance background interferes with segregating the striped and checkerboard regions in an elementarrangement pattern when the two types of squares composing a pattern differ primarily in hue. The two experiments reported here are investigations of how the lu- 
minances and hues of the interelement spaces between the squares, the luminance of the surround, the spatial separation of the squares, and the hues of the squares affect perceived segregation.

\section{GENERAL METHOD}

\section{Stimuli and Apparatus}

The stimuli were generated with a Symbolics 3600 Lisp machine and displayed on a Tektronix 690 SR RGB monitor. A pattern consisted of 15 rows and columns of squares of two different hues, as illustrated in Figure 1. In the top (rows 1-5) and bottom (rows 11-15) regions, the squares differing in hue were arranged in alternating vertical columns. In the center region (rows 6-10), the squares differing in hue were arranged in a checkerboard pattern. The squares were $0.36 \mathrm{in}$. ( 16 pixels) on a side and subtended approximately $17.3^{\prime}$ of arc. The hues of the squares, their spatial separation, the luminance of the surround, and the hue and luminance of the interspaces between the squares are reported below in the sections describing the individual experiments.

The luminances were measured with a Spectra-Pritchard brightness meter. The luminances of the squares differing in hue were equated to minimize perceived segregation due to luminance differences. Observations indicated that perceived segregation either remained the same or increased when the luminance of one of the hues composing a stimulus was either increased or decreased from the measured equal-luminance values

\section{Segregation Ratings}

The subjects were presented with four blocks of trials. On each block of trials, the order of the stimuli was randomized. The sub-

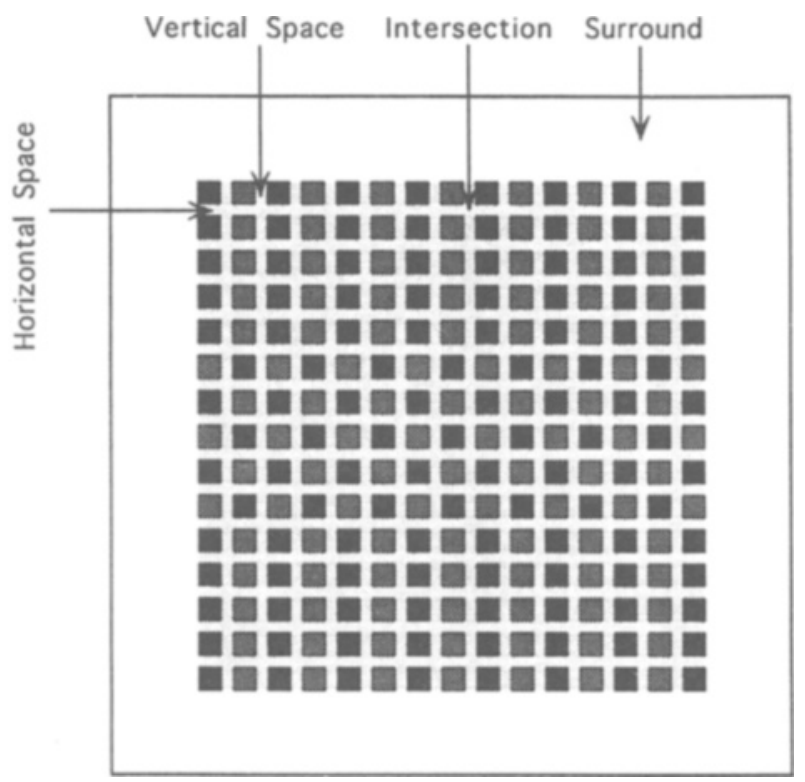

Figure 1. Illustration of the stimulus displays. The black and dark gray squares represent two different equal-luminance hues, such as red and blue. The interspace area is shown as light gray. Shown is the horizontal space between the squares, the vertical space between the squares, and the intersection area. Three different luminance distributions were investigated: the entire interspace luminance was varied (i.e., the luminances of the horizontal spaces, vertical spaces, and intersections), the luminance of the horizontal intersections was varied (i.e., the luminances of the horizontal spaces and of the intersections), and the luminance of the vertical intersections was varied (i.e., the luminances of the vertical spaces and the intersections). jects were instructed to base their judgments on their immediate impressions and not to scrutinize the patterns to find the boundaries between the three regions. They rated the perceived segregation of the striped and checkerboard regions on a 9-point scale, from 0 to 4 in steps of .5. A rating of 4 indicated that the regions appeared to be strongly segregated. A rating of 0 indicated that the regions did not appear to be segregate at all - that is, they appeared to constitute a single pattern. Control stimuli, in which all the squares in a display were the same hue, were included. The subjects were instructed to rate these displays as 0 . They were cautioned, however, that a rating of 0 should not be restricted to displays in which all the squares had the same hue, but should also be assigned when the two squares composing a display were readily discriminable but the display as a whole did not segregate into three regions. A display made up of Ts and Ls was presented as an example; the individual figures were readily discriminable, but the display failed to segregate into three regions (see Figure 12 in Beck, Prazdny, \& Rosenfeld, 1983). The subjects were shown sample stimuli to familiarize them with the range of stimulus variations, and they were given a practice block of trials. The subjects' mean segregation ratings are plotted in Figures 2-9.

A subject initiated a trial by pressing a mouse button. A trial consisted of a fixation "X," which was presented for $1 \mathrm{sec}$ in the center of a gray screen. This was followed immediately by a stimulus display, presented for $1 \mathrm{sec}$. A rating scale presented on a gray background immediately followed the offset of the stimulus display. A subject rated the perceived segregation by moving the mouse to highlight a number on the scale. The number was recorded by pressing the mouse button.

\section{Subjects}

Ten subjects participated in each of two experiments. Five of the subjects participated in both experiments. All of the subjects had normal or corrected-to-normal vision and were paid to participate. They were naive concerning the purpose of the experiments.

\section{EXPERIMENT 1}

Experiment 1 was an investigation of how the intensity and distribution of the luminances in the interelement spaces affected perceived texture segregation with a low-luminance (black) and high-luminance (white) surround.

\section{Stimuli}

The hues of the squares were red and blue and were set at $2.3 \mathrm{fL}$. The CIE coordinates for the red and blue hues were $x=.610, y=$ .340 , and $x=.152, y=.063$, respectively. There were 60 experimental stimuli: 3 experimental conditions, 5 luminances of the interspace area, 2 surround luminances, and 2 spacings of the squares. The hue of the intersquare interspaces was achromatic. The distribution of luminances in the interspace area was varied among conditions. As is shown in Figure 1, the interspace area consisted of the horizontal and vertical spaces between the squares and the intersection areas. In Condition 1, the luminance of the entire interspace was varied; that is, the luminances of the horizontal and vertical spaces between the squares and of the intersections varied (hereafter referred to as the luminance of the entire interspace). In Condition 2, the luminances of the horizontal spaces between the squares and the intersections were varied (hereafter referred to as the luminance of the horizontal interspaces). In Condition 3, the luminances of the vertical spaces between the squares and the intersections were varied (hereafter referred to as the luminance of the vertical interspaces). The luminances of the vertical spaces between the squares in Condition 2 and the horizontal spaces between the squares in Condition 3 were $.1 \mathrm{fL}$. The luminances of the interspace areas were $.1,2.3,4.4,7.0$, and 
$28.3 \mathrm{fL}$. The surround luminances were $1 \mathrm{fL}$ and $28.3 \mathrm{fL}$. The center-to-center spacings of the squares were 22 and 28 pixels.

\section{Results}

Figure 2 shows perceived segregation, with a spacing of 22 pixels and a black surround, as a function of luminance for the three luminance distributions. Figure 3 shows the same functions for the 28-pixel separation of the squares, and Figures 4 and 5 show the same functions for the 22- and 28-pixel separations of the squares with a white surround. The main effects of the distribution of luminance in the interspace area $[F(2,18)=$ $60.10, p<.05]$, luminance of the interspace area $[F(4,36)$ $=81.90, p<.05]$, surround luminance $[F(1,9)=39.95$, $p<.05]$, and square spacing $[F(1,9)=178.06, p<.05]$ were significant. Perceived segregation in all instances decreased with increasing luminance of the interspace area and with increasing spacing of the squares. A comparison of Figures 2 and 3 with Figures 4 and 5 shows that perceived segregation was decreased by a white surround, but the pattern of decrease in perceived segregation with increasing luminance was similar to that with a black surround.

Three interactions are of interest. The distribution of luminance in the interspace area $\times$ luminance of the interspace area interaction was significant $[F(8,72)=$

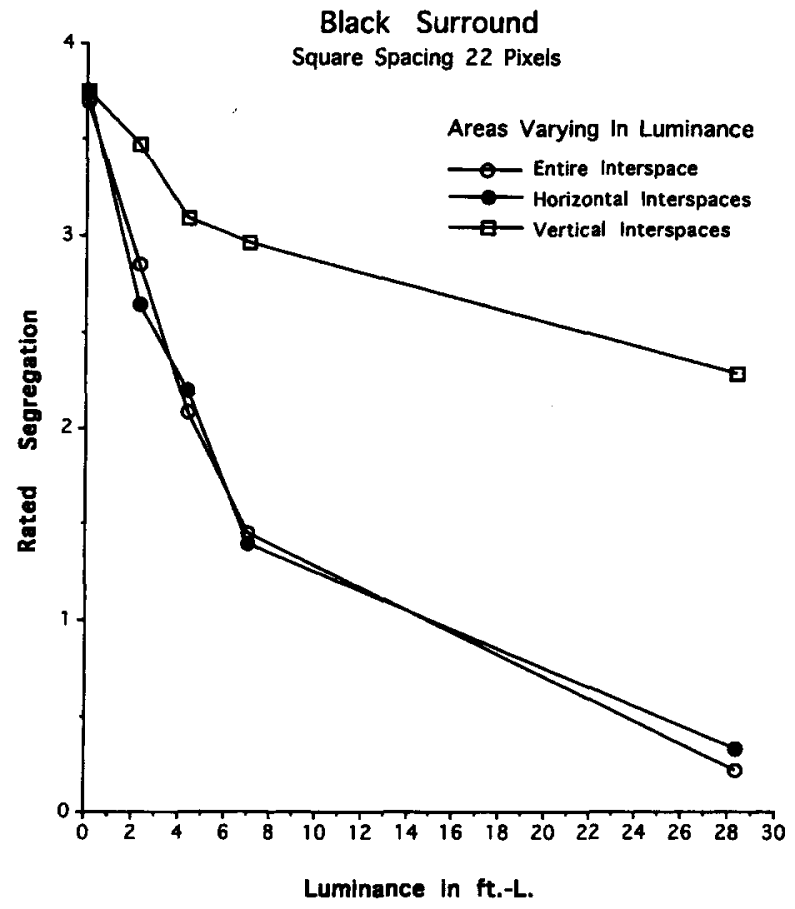

Figure 2. Mean segnegation ratings plotted as a function of luminance in Experiment 1. The center-to-center spacing of the squares was 22 pixels, and the surround was black. The results shown are for red and blue squares set at $2.3 \mathrm{fL}$ when the luminances of the interspace, the horizontal interspaces between the rows of a texture pattern, and the vertical interspaces between the columns of a texture pattern were varied.

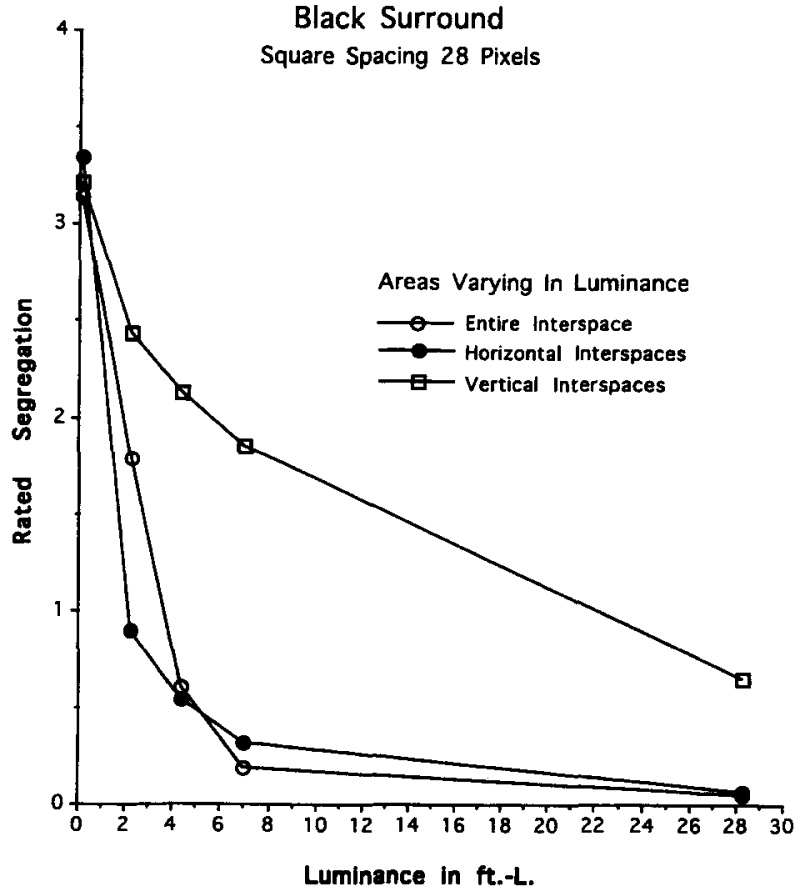

Figure 3. Mean segregation ratings plotted as a function of luminance in Experiment 1. The center-to-center spacing of the squares was 28 pixels, and the surround was black. The results shown are for red and blue squares set at $2.3 \mathrm{fL}$ when the luminances of the interspace, the horizontal interspaces between the rows of a texture pattern, and the vertical interspaces between the columns of a texture pattern were varied.

21.47, $p<.05]$. Perceived segregation decreased more and similarly when either the luminance of the entire interspace or the luminance of the horizontal interspaces was increased than when the luminance of the vertical interspaces was increased. The luminance of the interspace area $\times$ square spacing interaction was also significant $[F(4,36)=4.95, p<.05]$. This interaction shows that the interference of increasing the luminance in the interspace area decreased perceived segregation more with a 28-pixel spacing than with a 22-pixel spacing. The greater effect of the interspace area luminance with the larger intersquare spacing indicates a contrast $x$ area tradeoff on perceived segregation. The surround luminance $\times$ square spacing interaction, in contrast, was not significant $[F(1,9)=.07, p>.05]$. This indicates that the high-luminance surround had an overall depressive effect on perceptual segregation that was similar for the 22- and 28-pixel spacings of the squares.

\section{EXPERIMENT 2}

Experiment 2 was an investigation of whether the decrease in perceived segregation that occurred in Experiment 1 would be obtained with different hues of the squares and the interspace. The hues of the squares and the hue and luminance of the entire interspace area were varied. The surround was black. 


\section{Stimuli}

The luminances of the squares were $2.3 \mathrm{fL}$. The surround luminance was $.1 \mathrm{fL}$. There were 54 experimental stimuli. The hues of the squares were red and blue, red and green, and green and blue. The CIE coordinates of the red and blue hues were the same as those in Experiment 1. The CIE coordinates of the green hue were $x=.280, y=.590$. The center-to-center distances of the squares were 22 and 28 pixels. There were 22 stimuli in which the squares were red and blue: 11 with a 22-pixel center-to-center spacing, and 11 with a 28-pixel center-to-center spacing. For each spacing, the hue of the interspace was achromatic for 6 stimuli and green for 5 stimuli. There were 20 stimuli in which the squares were green and blue: 10 with a 22 -pixel center-to-center spacing and 10 with a 28 pixel center-to-center spacing. For each spacing, the hue of the interspace was achromatic for 6 stimuli and red for 4 stimuli. There were 12 stimuli in which the squares were red and green: 6 with a 22-pixel center-to-center spacing, and 6 with a 28-pixel center-to-center spacing. The hue of the interspace was achromatic. The luminances of the achromatic interspace were $.1,2.3$, $4.4,7.0,18.2$, and $28.3 \mathrm{fL}$, those of the green interspace were .1 , $2.3,4.4,7.0$, and $18.2 \mathrm{fL}$ (the maximum green luminance), and the luminances of the red interspace were $.1,2.3,4.4$, and $7.0 \mathrm{fL}$ (the maximum red luminance)

\section{Results}

Figures 6 and 7 show the perceived segregation of the red and blue, red and green, and blue and green squares when the interspace was achromatic, with 22- and 28pixel spacings of the squares. The main effects of interspace luminance $[F(5,45)=105.37, p<.05]$, square hue $[F(2,18)=16.29, p<.05]$, and square spacing $[F(1,9)=$ $164.67, p<.05]$ were significant. Perceived segregation

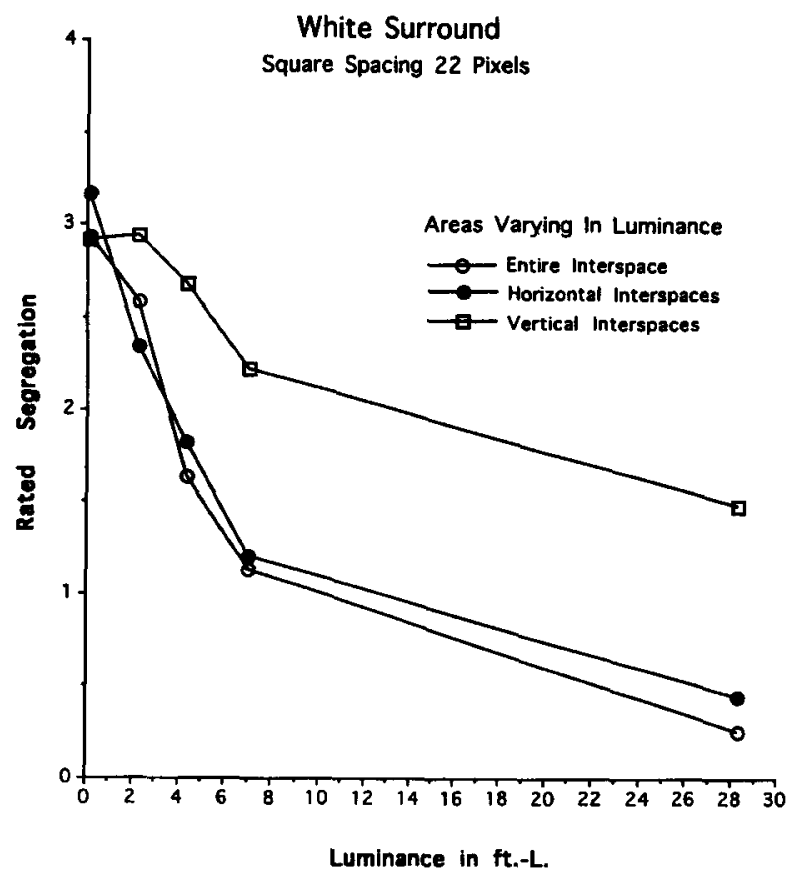

Figure 4. Mean segregation ratings plotted as a function of luminance in Experiment 1. The center-to-center spacing of the squares was 22 pixels, and the surround was white. The results shown are for red and blue squares set at $2.3 \mathrm{fL}$ when the luminances of the interspace, the horizontal interspaces between the rows of a texture pattern, and the vertical interspaces between the columns of a texture pattern were varied.

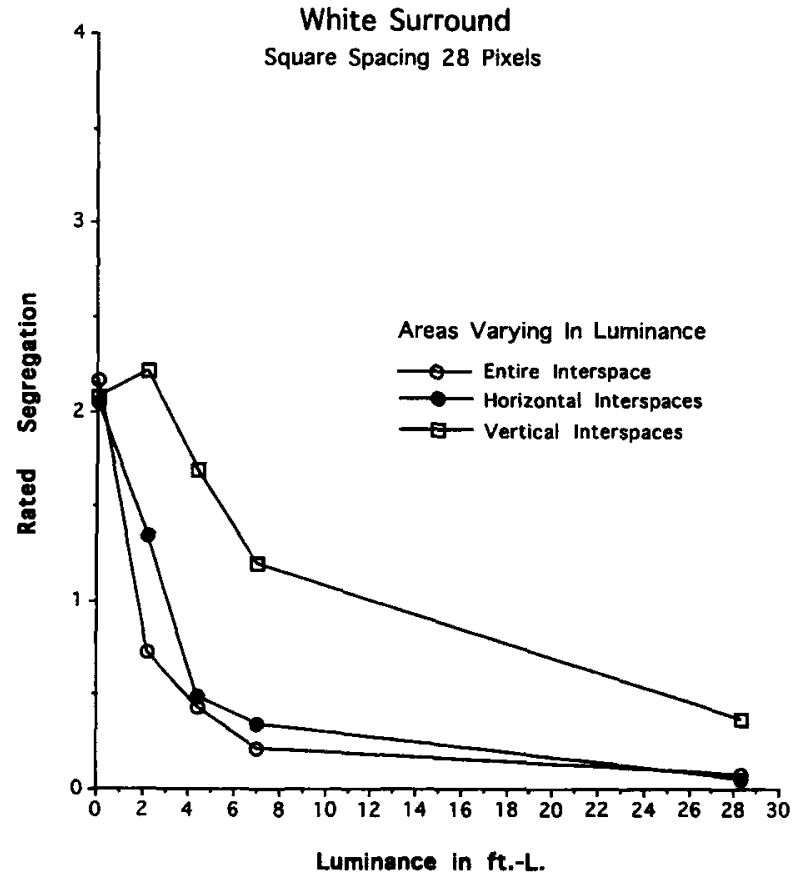

Figure 5. Mean segregation ratings plotted as a function of luminance in Experiment 1 . The center-to-center spacing of the squares was 28 pixels, and the surround was white. The results shown are for red and blue squares set at $2.3 \mathrm{IL}$ when the luminances of the interspace, the horizontal interspaces between the rows of a texture pattern, and the vertical interspaces between the columns of a texture pattern were varied.

in all cases decreased with increased interspace luminance and with increased spacing of the squares. Also, the red and blue squares segregated more strongly than the red and green and blue and green squares. The differences in the perceived segregation between the red and blue and the red and green squares $[F(1,9)=55.65$, $p<.05]$ and between the red and blue and the blue and green squares $[F(1,9)=21.99, p<.05]$ were significant. The differences in the perceived segregation of the red and green and the blue and green squares were not significant $[F(1,9)=.05, p>.05]$. An area $\times$ contrast tradeoff was again found. The interaction of interspace luminance $\times$ square spacing was significant $[F(5,45)=$ $9.64, p<.05]$. Increasing luminance interfered less when the square spacing was 22 pixels than when it was 28 pixels.

Figure 8 shows perceived segregation as a function of the luminance of the achromatic and green interspaces with 22- and 28-pixel spacings. Perceived segregation of the red and blue squares decreased with increased luminance of the interspace $[F(4,36)=134.54, p<.05]$, but was not affected by whether the interspace was achromatic or green $[F(1,9)=.16, p>.05]$. The interaction of interspace luminance $\times$ square spacing was significant $[F(4,36)=19.27, p<.05]$, indicating that the interference of increasing interspace luminance was greater with a 28-pixel spacing. In contrast, the perceived segregation of the blue and green squares was affected by 


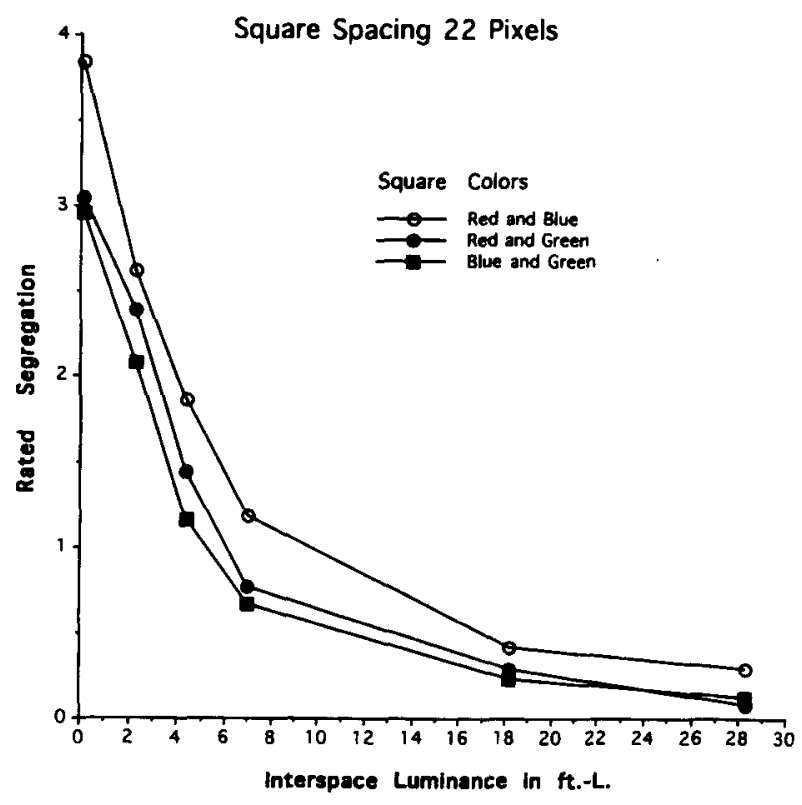

Figure 6. Mean segregation ratings plotted as a function of the luminance of the interspace in Experiment 2. The results shown are for the red and blue, red and green, and blue and green squares set at $2.3 \mathrm{fL}$. The center-to-center spacing of the squares was 22 pixels, and the surround was black.

whether the interspace was achromatic or red. Figure 9 shows perceived segregation as a function of the luminances of the achromatic and red interspaces, with 22and 28-pixel spacings of the squares. Perceived segregation decreased with increasing luminance $[F(3,27)=$ $65.30, p<.05]$ and was greater with an achromatic interspace than with a red interspace $[F(1,9)=23.43, p<.05]$. The interaction of interspace luminance $X$ square spacing was not significant $[F(3,27)=2.27, p>.05]$.

The reason for the difference in perceived segregation due to the hues of the squares and the interspaces is unclear. It may be due to hue interactions. The point to note is that increasing the interspace luminance decreased perceived segregation for all of the patterns, irrespective of square and interspace hue.

\section{DISCUSSION}

The results show that in element-arrangement patterns composed of squares of equal size and luminance but of two different hues, (1) perceived segregation decreased with increasing luminance of the interspace areas; (2) increasing the luminance of the horizontal interspaces decreased perceived segregation more than increasing the luminance of the vertical interspaces; (3) perceived segregation was less with a high-luminance surround than with a low-luminance surround; and (4) increasing the spacing of the squares decreased perceived segregation.

Beck, Graham, and Sutter (1991) found that the perceived segregation in an element-arrangement pattern was not determined by the lightness difference of the squares composing the pattern. Large differences in lightness sometimes failed to yield perceptual segregation. Perceived segregation was largely, but not completely, determined by the ratio of the luminance contrasts of the squares. The perceived segregation was ascribed to the differences in the outputs of simple-celllike receptive fields that are sensitive to the fundamental spatial frequency and orientation of the texture region defined by the arrangement of the squares (Beck et al., 1991). In the present experiments, the luminance contrasts of the squares provided no basis for segregation, since the luminances of the squares were equal. Instead, perceived segregation was based on the differences in the hues of the squares. For low-luminance interspaces, the perceived segregation of the striped and checkerboard regions was strong; for high-luminance interspaces, the perceived segregation of the striped and checkerboard regions either did not occur, or was very weak. These results are similar to those that occur with achromatic squares differing in lightness (Beck et al., 1991). It seems likely that the underlying reasons for the interference of high-luminance interspaces in segregating the striped and checkerboard regions are similar for element-arrangement patterns composed of squares differing in lightness and squares differing in hue.

The results admit two kinds of explanation. One possibility is that perceived segregation is affected by inhibitory interactions among cortical filters tuned to spatial frequency and orientation. Wavelength-selective simple and complex cells that are oriented have been reported by investigators (Michael, 1985; Ts'o, 1989). A set of multiple, wavelength-selective, spatial-frequency,

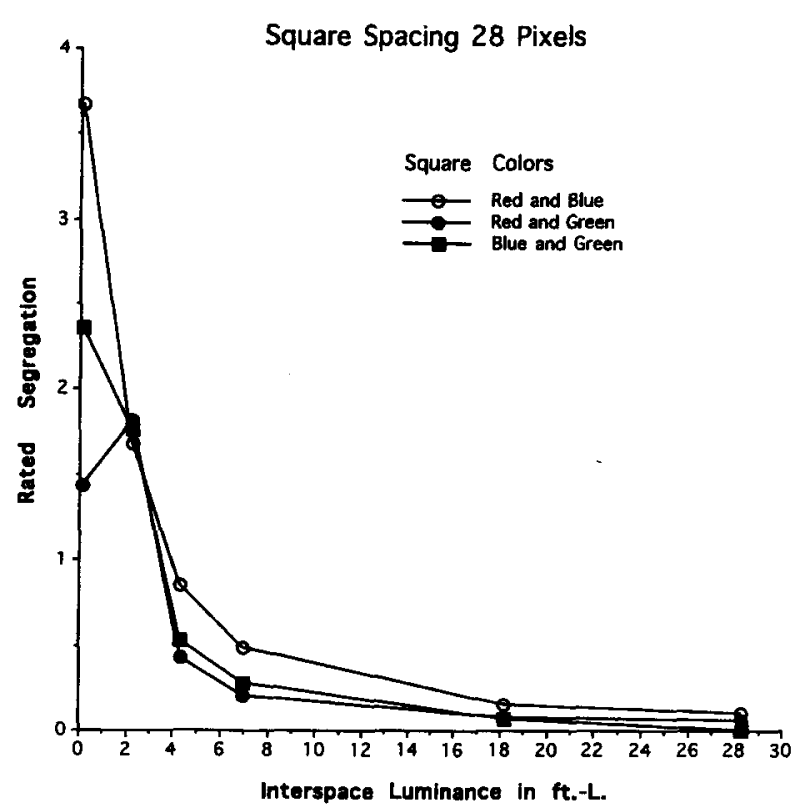

Figure 7. Mean segregation ratings plotted as a function of the luminance of the interspace in Experiment 2. The results shown are for red and blue, red and green, and blue and green squares set at $2.3 \mathrm{fL}$. The center-to-center spacing of the squares was 28 pixels, and the surround was black. 
and orientation-selective receptive fields would respond differently to the striped and checkerboard regions. Wavelength-selective oriented receptive fields whose excitatory region coincides with a column of squares would, for example, respond strongly to the vertical columns of red and blue squares in the top and bottom striped regions and to the diagonal columns of squares in the center region. One needs to further assume that the wavelength-selective oriented receptive fields that provide information for the difference in the arrangement of the red and blue squares in the striped and checkerboard regions are inhibited by the vertical- and horizontal-broadband receptive fields that respond to the interspace luminance. Since increasing the luminance of the horizontal interspaces (i.e., the lines perpendicular to the vertical columns of squares) interferes with perceptual grouping more than increasing the luminance of the vertical interspaces (i.e., the lines parallel to the vertical columns of squares), the data require that there be greater inhibition from the broadband, orthogonal, horizontal receptive fields than from the broadband, parallel, vertical receptive fields. The fact that surround luminance did not interact with spatial separation suggests that the depressive effect of a highluminance surround has a different basis. The decrease in perceptual grouping with a high-luminance surround may result from the hues' becoming more similar as a consequence of their darkening by contrast.

A second possibility is that the high-luminance interspaces interfere with perceptual grouping processes. Perceived texture segregation also occurs as a result of preattentive grouping operating on elementary proper-

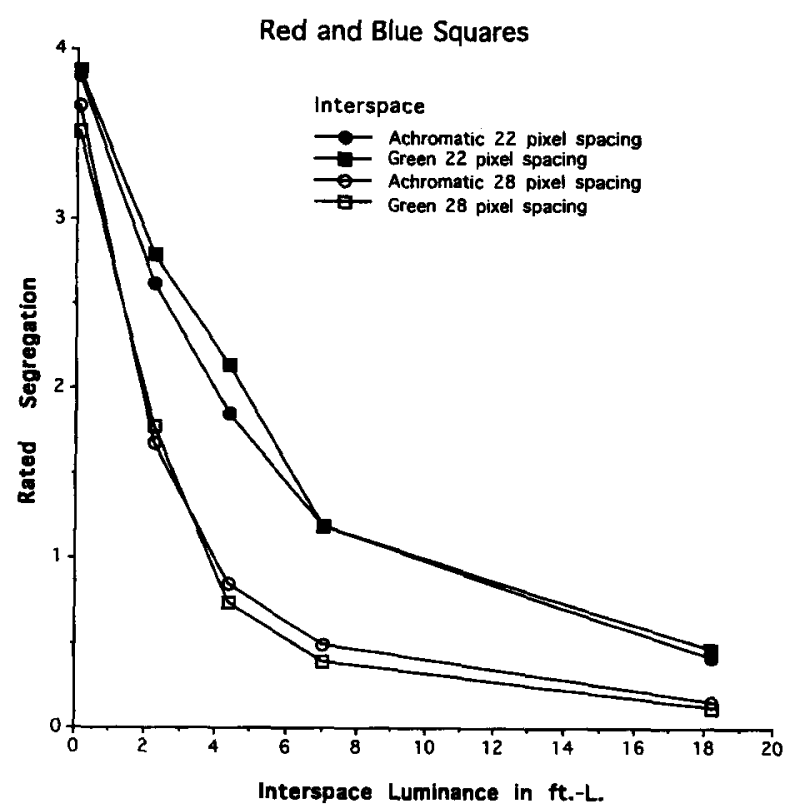

Figure 8. Mean segregation ratings plotted as a function of the luminance of the interspace in Experiment 2. The results shown are for red and blue squares set at $2.3 \mathrm{fL}$ with white and green interspace hues. The center-to-center spacings of the squares were 22 and 28 pixels, and the surround was black.

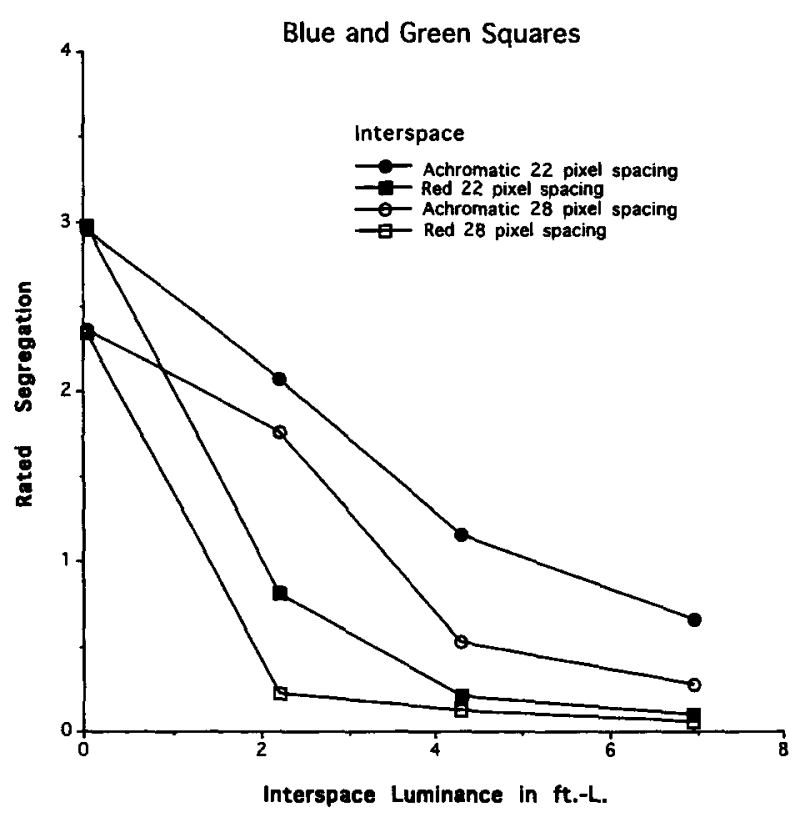

Figure 9. Mean segregation ratings plotted as a function of the luminance of the interspace in Experiment 2. The results shown are for blue and green squares set at $2.3 \mathrm{fL}$ with white and red interspace hues. The center-to-center spacings of the squares were 22 and 28 pixels, and the surround was black.

ties, such as edges and lightnesses. Perceived segregation has been shown to occur as a result of the grouping of collinear edges (Beck, 1983; Beck et al., 1983; Beck, Rosenfeld, \& Ivry, 1989) and of edges that form continuous curves (Field, Hayes, \& Hess, 1993). Perceived segregation in these instances appears to be the result of grouping processes that operate on the outputs of the receptive fields, which respond to the edges of the elements composing a pattern. Perceived segregation also occurs as the result of the similarity grouping of intermixed light and dark squares into two subpopulations of light and dark squares. Perceived segregation is in accord with the perceived lightness differences of the squares, and it appears to be the result of grouping processes operating after the lightnesses of the squares are computed (Beck et al., 1991). Experiments with space-averaged luminance and chromatic elements indicate that lightness and hue grouping occur not in terms of interactions among cortical filters selective for luminance and wavelength, but in terms of visual attributes such as hue and lightness, which are computed after the visual image has been chunked into individual elements (Beck \& Goodwin, 1992). Grouping processes also appear to be the basis of perceptual segregation that results from the three-dimensional interpretation of projected shapes (Beck, 1993). Perceptual segregation based on the three-dimensional interpretation of projected shapes, however, differs fundamentally in that it requires attention (Beck, 1993; Perez-Martinez, 1992).

The arrangement of the squares differing in hue in an element-arrangement pattern allows for the squares in the top and bottom regions to be grouped into vertical 
columns. In contrast, the checkerboard arrangement of the squares in the middle region allows for the diagonal grouping of the squares. These different groupings of the squares lead to edge-like discontinuities at the boundaries of the striped and checkerboard regions and facilitate perceived segregation. The greater effectiveness of the luminance of the horizontal interspaces than that of the vertical interspaces in decreasing perceived segregation suggests that interspace luminance interferes with perceived segregation by interfering with the grouping of the squares based on hue into vertical columns in the top and bottom striped regions of the pattern. However, the nature of the grouping processes and the interference is unclear. In accordance with proximity grouping, this interference is greater for small spatial separations of the squares than for large spatial separations of the squares. In general, however, there is no interference of proximity grouping by high-luminance interspaces. The results are also not consistent with pure similarity grouping. An example of pure similarity grouping is the grouping of intermixed light and dark squares into two subgroups. Perceptual segregation of the intermixed light and dark squares into two subgroups was primarily a function of the lightness differences of the squares and was not greatly affected by the distances between the squares or by the luminances of the interspaces (Beck et al., 1991). It is worth noting that observations show that the interference of the high-luminance interspaces does not depend on the alignment of the squares. When the squares differing in hue in an element-arrangement pattern are jittered, the results are the same as those found when the squares are aligned. Grossberg and Mingolla (1985), using competitive-cooperative feedback interactions, have explained the grouping of contours and lines in elementarrangement patterns. A comparable model explaining how local image attributes, such as hues, are grouped in an element-arrangement pattern remains to be developed.

\section{REFERENCES}

BECK, J. (1983). Textural segmentation, second-order statistics, and textural elements. Biological Cybernetics, 48, 125-130.

BECK, J. (1993). Visual processing in texture segregation. In D. Brogan, A. Gale, \& K. Carr (Eds.), Visual search 2 (pp. 1-35). London: Taylor \& Francis.
BECK, J., \& Goodwin, W. (1992). Prevailing lightness and hue and perceived texture segregation. In G. Carpenter \& S. Grossberg (Eds.), Neural networks for vision and image processing (pp. 15-43). Cambridge, MA: MIT Press.

Beck, J., Graham, N., \& Sutter, A. (1991). Lightness differences and the perceived segregation of regions and populations. Perception $\&$ Psychophysics, 49, 257-269.

Beck, J., Prazdny, K., \& Rosenfeld, A. (1983). A theory of textural segmentation. In J. Beck, B. Hope, \& A. Rosenfeld (Eds.), Human and machine vision (pp. 1-38). New York: Academic Press.

BECK, J., RosenFELD, A., \& IVRY, R. (1989). Line segregation. Spatial Vision, 4, 75-101.

Beck, J., SutTer, A., \& IVRY, R. (1987). Spatial-frequency channels and perceptual grouping in texture segregation. Computer Vision, Graphics, \& Image Processing, 37, 299-325.

Bergen, J. R., \& LANDY, M. S. (1991). Computational modeling of visual texture segregation. In M. S. Landy \& J. A. Movshon (Eds.), Computational models of visual processing (pp. 253-271). Cambridge, MA: MIT Press.

FIELD, D. J., HAYES, A., \& Hess, R. F. (1993). Contour integration in the human visual system: Evidence for a "local association field." Vision Research, 33, 173-193.

FoGEL, I., \& SAGI, D. (1989). Gabor filters as texture discriminator. Biological Cybernetics, 61, 103-113.

Graham, N., Beck, J., \& SutTer, A. (1992). Nonlinear processes in spatial-frequency channel models of perceived texture segregation: Effects of sign and amount of contrast. Vision Research, 32, 719-743.

Grossberg, S., \& Mingolla, E. (1985). Neural dynamics of perceptual grouping: Textures, boundaries, and emergent segmentations. Perception \& Psychophysics, 38, 141-171.

Malik, J., \& Perona, P. (1990). Preattentive texture discrimination with early vision mechanism. Journal of the Optical Society of America A, 2, 923-932.

MichaEL, C. R. (1985). Serial processing of color in the monkey's striate cortex. In D. Rose \& V. G. Dobson (Eds.), Models of the visual cortex (pp. 301-309). London: Wiley.

Perez-MARTINeZ, D. (1992, September). Insensitivity to monocular cues of tridimensionality in preattentive texture discrimination. Paper presented at the Third International Conference on Visual Search, York, England.

SutTer, A., BeCK, J., \& Graham, N. (1989). Contrast and spatial variables in texture segregation: Testing a simple spatial-frequency channels model. Perception \& Psychophysics, 46, 312-332.

Ts'o, D. (1989). The functional organization and connectivity of color processing. In D. Man-Kit Lam \& C. D. Gilbert (Eds.), Neural mechanism of visual perception (pp. 87-115). Woodlands, TX: Portfolio Publishing.

TuRNER, M. R. (1986). Texture discrimination by Gabor functions. Biological Cybernetics, 55, 71-82.

(Manuscript received July 6, 1993; revision accepted for publication March 30, 1994.) 